\section{Multiple opponents}

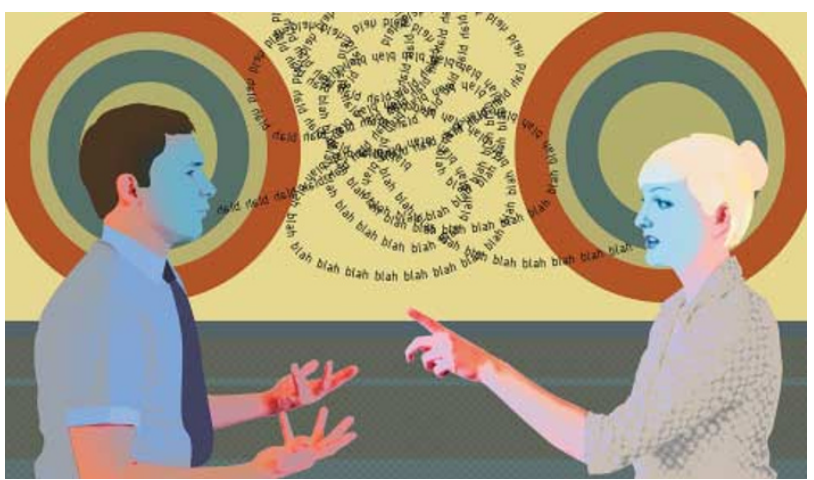

Patients with chronic myelogenous leukaemia, acute lymphoblastic leukaemia and gastrointestinal tumours treated long term with imatinib (Glivec) almost always develop acquired resistance. Several mechanisms of imatinib resistance have recently been documented and now Burger and colleagues have identified yet another novel mechanism mediated by the breast cancer resistance gene $B C R P$.

Imatinib, a tyrosine-kinase inhibitor effective against BCR-ABL1, KIT and platelet-derived growth factor receptor, is an orally administered drug. Given that BCRP is highly expressed within the gastrointestinal tract and is a member of the ABC family of drug transporters, it seems likely that BCRP is involved in limiting the uptake of imatinib into the blood, thereby limiting the biological availability of imatinib. So, Burger et al. asked if imatinib is a substrate of BCRP. Initially, the authors used two breast cancer cell sublines that overexpressed either wild-type (Arg482) or mutant (Thr482) BCRP, a mutation previously shown to affect substrate specificity. Carbon-14 labelled imatinib was used to measure drug uptake. Both wild-type overexpressing cells and mutant-BCRP expressing cells had significantly less drug accu- mulation than controls, indicating that both the mutant and wild-type BCRP proteins act as drug-efflux pumps for imatinib. Real-time PCR analyses and western-blot data confirmed that other $\mathrm{ABC}$ drug-efflux proteins - MDR1, MRP1 and MRP2 - were not overexpressed in these cells.

To further examine this interaction, the authors monitored the efflux of imatinib and three other known BCRP substrates: mitoxantrone, and methotrexate and doxorubicin two substrates that are exclusive targets for wild-type or mutant BCRP, respectively. Imatinib is exported in a similar manner to mitoxantrone by both wild-type- and mutant-BCRP expressing cells. In addition, efflux of imatinib was reduced in the presence of a BCRP-specific inhibitor, further demonstrating that imatinib is a bona fide substrate for BCRP.

The similar efflux profiles of imatinib and mitoxantrone indicated that imatinib might be a competitive

\section{CANCER SUSCEPTIBILITY}

\section{$\mathrm{X}$ marks the spot}

Susceptibility to cancer is one of the serious consequences of Fanconi anaemia (FA), an autosomal recessive disease that causes congenital abnormalities, progressive bonemarrow failure and hypersensitivity to DNAcrosslinking agents. Several of the defective proteins responsible for the disease are normally involved in repairing DNA damage, and a newly described FA-associated polypeptide - FAAP95, identified by Hans Joenje and colleagues - is no exception. What is unexpected is that the gene encoding FAAP95 underlies complementation group B (FA-B), which was previously thought to be associated with BRCA2. Furthermore, the gene encoding FAAP95 is X-linked, a discovery that brings with it profound implications for carriers of the defective gene as well as new insights into sporadic cancer.

The researchers identified FAAP95 by analysing components of the FA 'core complex', which is responsible for the monoubiquitylation of a key protein involved in the repair of DNA crosslinks, FANCD2. They found that the depletion of FAAP95 reduced the amount of monoubiquitylated
FANCD2 produced, prompting the team to examine whether the gene encoding FAAP95 might be one of those responsible for FA. Having obtained the amino-acid sequence of FAAP95, a database search showed that the gene encoding it is localized on the $\mathrm{X}$ chromosome. The researchers then looked for a FA complementation group in which all affected individuals were male and had defective monoubiquitylation. Of the 11 identified complementation groups, only one fulfilled these criteria: FA-B. When the researchers sequenced the region encoding FAAP95 in FA-B individuals, they found frameshift mutations that cause premature stop codons. They also transfected FA-B lymphoblasts with cDNA encoding wild-type FAAP95, and found that monoubiquitylation and other phenotypes were restored to normal, confirming that FAAP95 was defective in FA-B individuals. The gene was therefore named $F A N C B$.

$\mathrm{X}$-linked inheritance of FA has not previously been reported, and this prompted the researchers to test mothers and grandmothers of FA-B individuals to see if they carried mutant FANCB. Surprisingly, although female carriers were identified, some of the patients' mothers did not seem to carry the mutations. By examining the methylation state of FANCB in these kindreds, the researchers found that mutant
FANCB in fact tended to be subject to $\mathrm{X}$-inactivation - whereby most genes on one of the two X-chromosomes are transcriptionally silenced during early embryogenesis. The authors speculate that during development, healthy cells, in which mutant FANCB is inactive, out-compete cells expressing mutant copies of the gene, resulting in carriers with normal FANCB in most of their tissues.

Nevertheless, the possibility that carriers might have some FA-like tissues means that even apparently healthy relatives of individuals with X-linked FA are at risk from malignancies, and the authors emphasize the importance of monitoring FANCB carriers for cancer. Furthermore, somatic inactivation of the FA pathway accounts for some cancers within the general population. The researchers warn that $\mathrm{X}$-linked $F A N C B$ is a particularly vulnerable component of the FA-BRCA DNA-damage pathway that could fall foul of the spontaneous mutation rate in somatic cells.

Jenny Bangham

(D) References and links

ORIGINAL ReSEARCH PAPER Meetei, A. R et al. X-linked inheritance of Fanconi anemia complementation group B. Nature Genet. 11, 1219-1224 (2004)

FURTHER READING D'Andrea, A. D. \& Grompe, M. The Fanconi anaemia/BRCA pathway. Nature Rev. Cancer $\mathbf{3}$ 23-34 (2003)

Hans Joenje's lab:

http://www.niwi.knaw.nl/en/oi/nod/onderzoeker/PRS1252392/ 